\title{
Anpassungsprozesse beim Übergang in die Sekundarstufe I
}

Markus P. Neuenschwander

\section{Zusammenfassung}

Die wahrgenommene Passung zwischen Unterrichtsangeboten und Bedürfnissen bzw. Vorwissen der Kinder begünstigt Lern- und Entwicklungsprozesse. Aufgrund der Veränderungsprozesse wird vermutet, dass sich beim Übertritt in die Sekundarstufe I die Passungswahrnehmung, aber auch die Motivations- und Leistungsindikatoren, verschlechtern. In der Längsschnittstudie Wirkungen der Selektion (WiSel) wurden 498 Kinder im 5., 6. und 7. Schuljahr aus 4 Kantonen mit Übertritt nach dem 5. bzw. 6. Schuljahr befragt. Kovarianzanalysen bestätigen die Hypothesen: Passungswahrnehmung, Lern- und Leistungszielorientierung, Noten in Deutsch und Mathematik nehmen bei einem frühen Übertritt in die Sekundarstufe I stärker ab als bei einem späten Übertritt. Die Leistungszunahme in Leistungstests in Deutsch und Mathematik ist beim Übertritt nach dem 5 . Schuljahr geringer als beim Übertritt nach dem 6. Schuljahr. Beim Übertritt korreliert die Stärke der Abnahme der Passungswahrnehmung mit der Veränderung der Noten und der Lern- und Leistungsziele. In Übereinstimmung mit Eccles et al. (1993) wird vermutet, dass sich die Schulorganisation der Sekundarstufe I mit verschiedenen Leistungsniveaus, mit einem Fachlehrpersonensystem und mit stufenspezifischen Lehrplankonzepten weniger günstig auf die Motivations- und Leistungsindikatoren auswirkt als diejenige der Primarstufe ohne Leistungsniveaus und Klassenlehrpersonensystem.

\section{Schlagworte}

Transition, Passung, Leistungen, Noten, Lernzielorientierung, Leistungszielorientierung, Sekundarstufe I 


\section{Ausgangslage}

Der Übertritt in die Sekundarstufe I ist in den Deutschschweizer Kantonen eine normative Schultransition, bei der Kinder in einem standardisierten Selektionsprozess einem Schulniveau mit unterschiedlich hohen schulischen Anforderungen zugewiesen werden. Damit werden erste Entscheidungen für den Bildungsverlauf und den Berufseintritt gefällt. Dieser Übertritt beginnt mit einem Selektionsprozess und endet mit einem Anpassungsprozess der Kinder. Während die Selektionsprozesse beim Übertritt in die Sekundarstufe I seit vielen Jahren intensiv untersucht worden sind (z. B. Maaz, Hausen, McElvany, Baumert, 2006; Neuenschwander \& Malti, 2009), wurden die Anpassungsprozesse nach Eintritt in die Sekundarstufe I, d.h. die Prozesse der Auseinandersetzung der Lernenden mit der neuen Situation in der Sekundarstufe I, weniger intensiv bearbeitet (z. B. Eccles et al., 1993). Dies ist erstaunlich, weil diese Anpassungsprozesse den weiteren Lern- und Entwicklungsprozess der Schülerinnen und Schüler wesentlich steuern. Diese Anpassungsprozesse werden im Folgenden ins Zentrum gestellt. Im vorliegenden Beitrag wird der Frage nachgegangen, wie sich Schülerinnen und Schüler nach dem Übertritt in die Sekundarstufe I an den neuen Kontext anpassen.

\section{$2 \quad$ Passung}

Frühere Forschung zeigte, dass eine hohe Passung zwischen dem Unterricht und den individuellen Bedürfnissen der Kinder eine hohe Motivation und hohe Leistungen in den Schulfächern begünstigt (Zimmer-Gembeck, Chipuer, Hanisch, Creed, McGregor, 2006; Eccles \& Roeser, 2009). Das Ausmass der Passung kann daher als Merkmal von Schulqualität gelten. Passung kann in Weiterführung zu Kristof-Brown, Zimmermann \& Johnson (2005) objektiv als Korrespondenz zwischen den schulischen Angeboten und dem individuellen Lernstand verstanden werden oder schülersubjektiv als wahrgenommene Korrespondenz zwischen dem Unterricht und den eigenen Bedürfnissen und Kompetenzen. Diese subjektive Passung, die im vorliegenden Beitrag im Zentrum steht, drückt sich in der Wahrnehmung aus, die eigenen Stärken im Unterricht gut einbringen zu können, motiviert zu sein, interessante Aufgaben zu erhalten und die eigenen Fähigkeiten zu verbessern. Eine hohe Passungswahrnehmung zwischen Person und Unterricht zeigt sich also darin, dass die Lehrangebote als auf die individuellen Fähigkeiten und Interessen abgestimmt wahrgenommen werden. 
Die Kontexte, welche Jugendliche in Bildungsverläufen durchschreiten, passen unterschiedlich gut zu ihrem Entwicklungsstand. Diese These wurde von Eccles et al. (1993; 2004) mit dem Konzept Stage-environment-fit im US-amerikanischen Raum umschrieben. Eccles (2004) meinte damit, dass die entwicklungsspezifischen Bedürfnisse, Interessen und Kompetenzen (stage) mit der schulischen Umwelt bzw. mit dem Unterricht (environment) korrespondieren sollten, damit sich das Kind optimal entwickeln kann. Diese Passung ist hoch, wenn die schulischen Angebote und Anforderungen optimal auf die alterstypische Situation passen.

Aus einer theoretischen Perspektive beschreibt Passung eine grundlegende Intelligenzleistung im Sinne von Piaget (1947) und Sternberg (1986), um das Überleben eines Menschen zu sichern. Intelligenz meint hier, dass sich Menschen so an eine Umwelt anpassen müssen, dass sie sich optimal entwickeln können. Bezogen auf den Schulkontext schliesst dies einerseits die Veränderung der eigenen Erwartungen an die Anforderungen der Schule, andererseits die Auswahl der passenden Schule sowie der Versuch, die Schule nach den eigenen Bedürfnissen zu gestalten, ein. Passung ist nach dieser Konzeption dynamisch und muss laufend neu hergestellt werden, weil sich sowohl das Individuum als auch die Schule ständig verändern. Mit Passung sind also nicht gemeint, dass Schülerinnen und Schüler konform und gehorsam sind und sich Autoritäten unterordnen.

Das Schaffen einer Passung ist aber auch eine Aufgabe der Schule bzw. des Unterrichts (Eccles et al., 1993). Lehrpersonen stehen im Spannungsfeld, einerseits die Ziele des Lehrplans zu verfolgen und sich andererseits an den Voraussetzungen der Schülerinnen und Schüler zu orientieren. Lehrpersonen unterrichten wirksam, wenn ihre Lernangebote auf die Bedürfnisse und Kompetenzen der Schülerinnen und Schüler abgestimmt sind (vgl. adaptiver Unterricht, Übersicht in Reusser, Pauli, Waldis, 2010). Lehrprozesse erzeugen maximalen Lernerfolg, wenn sie ideal an den Voraussetzungen der Schülerinnen und Schüler ansetzen. Entsprechend forderten Eccles et al. (1993), dass Schulen nicht ausschliesslich Bildung vermitteln, sondern ein Lernumfeld anbieten, das die entwicklungsspezifischen Bedürfnisse und das Wissen der Kinder berücksichtigt (Adaptivität).

\section{$3 \quad$ Lern- und Leistungszielorientierung, Leistung und Noten}

Neben der subjektiven Passungswahrnehmung sind auch Motivation und Leistungen Indikatoren für gelungene Anpassungsprozesse der Kinder an die schulischen Anforderungen. Im Folgenden wird auf die Lern- und Leistungsziele, die als Aspekte 
der Motivation verstanden werden und in den letzten Jahren hohe Aufmerksamkeit erhalten haben, fokussiert. Pintrich, Conley und Kempler (2003) beschrieben die Lern- und Leistungszielorientierung als kognitive Repräsentation davon, was Individuen leisten wollen. Sie sind im Unterschied zu Lebenszielen eher situationsund aufgabenspezifisch. Leistungszielorientierung wird oft in Lernziele (mastery goals) und Leistungsziele (performance goals) differenziert. Lernziele fokussieren auf den Erwerb von Kompetenzen und das Verstehen von Aufgaben. Leistungsziele beschreiben hingegen das Zeigen von Kompetenzen und das Überlegensein gegenüber anderen in der Gruppe (vgl. Neuenschwander, im Druck). Während die Korrelationen zwischen Wichtigkeit von Lernzielen (d.h. Lernzielorientierung) bzw. Leistungszielen (d.h. Leistungszielorientierung) und Leistungstestergebnissen bzw. Noten in der Regel klein sind oder fehlen, erklären Kombinationen von Lern- und Leistungszielorientierung im Jugendalter Leistungstestergebnisse und Noten bedeutsam (Conley, 2012). Die Lern- und die Leistungszielorientierung beschreiben also zwei Aspekte von leistungsrelevanten Einstellungen von Schülerinnen und Schülern, deren Kombination motivationale Haltungen ausdrückt und Leistungsunterschiede der Schülerinnen und Schüler erklärt.

Neben der Passungswahrnehmung und der Lern- und Leistungszielorientierung von Kindern bilden auch die Leistungen von Kindern in Deutsch und Mathematik Indikatoren für den Erfolg von Anpassungsprozessen. Leistungen beschreiben die Ergebnisse von fachspezifischen Leistungstests und sind von Noten, die Leistungsrückmeldungen von Lehrpersonen gegenüber den Kindern widerspiegeln und sich in der Regel am Leistungsniveau der Klasse abbilden, abzugrenzen (z. B. Neuenschwander, 2016). Es wird angenommen, dass Kinder bessere Leistungen in Deutsch und Mathematik erbringen und dadurch bessere Noten in Deutsch und Mathematik erreichen, wenn sie sich an die Angebote und Anforderungen im Unterricht anpassen können.

\section{$4 \quad$ Übertritt in die Sekundarstufe I}

Eccles (2004) berichtete aufgrund einer Analyse von US-amerikanischen Schulen, dass beim Übertritt in die Sekundarstufe I die Passung zwischen dem Kind und dem Unterricht abnimmt, weil die Beziehungen in grossen Schulen anonymer sind und weil die Leistungsanforderungen bei Eintritt in die Highschool zunehmen. Sie postulierte daher eine geringere Passung zwischen den altersspezifischen Bedürfnissen der Kinder und dem schulischen Umfeld in der Sekundarstufe I im Vergleich zur Primarschule. Entsprechend nimmt Eccles (2004) an, dass die 
Lern -und Leistungsorientierung während des Übertritts in die Sekundarstufe I besonders abnehmen und die Leistungszuwächse aufgrund der abnehmenden Passungswahrnehmung geringer werden. Diese These korrespondiert mit Befunden von Alspaugh \& Harting (1995), Alspaugh (1998), Whitley, Lupart \& Beran (2007) sowie van Ophuysen (2008).

Weil die Jugendlichen in der Schweiz mit dem Übertritt in die Sekundarstufe I in ein Schulniveau mit leistungshomogeneren Schulklassen übertreten, ist nicht klar, ob diese These auf die Schule in der Schweiz übertragen werden kann. In der Deutschschweiz treten die Kinder nach der Primarschule in die gegliederte Sekundarstufe I über. Die Kinder werden aufgrund ihrer Leistungen, Elternerwartungen und des Gesamteindrucks der Lehrperson einem Schulniveau zugewiesen. Die Selektionskriterien und der Übertrittszeitpunkt unterscheiden sich zwischen den Kantonen. Während in den Kantonen Aargau und Basel-Landschaft ${ }^{1}$ der Übertritt nach dem 5. Schuljahr stattfindet, vollzieht er sich in den Kantonen Bern und Luzern nach dem 6. Schuljahr.

Analog zur USA sind die Schweizer Schulen in der Sekundarstufe I in der Regel grösser und anonymer als diejenigen der Primarschule. Aufgrund des Fachgruppenlehrpersonensystems in der Sekundarstufe I rücken die persönlichen Beziehungen zwischen Lehrpersonen und Schülerinnen und Schülern im Vergleich zu den Inhalten eher in den Hintergrund. Die Schülerinnen und Schüler müssen sich in einer neuen Schulklasse eine Position/Stellung erwerben (Rösselet \& Neuenschwander, in diesem Band). In der Sekundarstufe I wird der Unterricht weniger individualisiert und stärker lehrerzentriert durchgeführt (Ludwig-Tauber, Wild-Naef, Vouets, 2000; Pauli, Reusser, Waldis, Grob, 2003; Götz, Lohmann, Ganser, Haag, 2005). Daher ist der Übertritt für die Kinder eine Herausforderung, wenn auch in der Regel keine Überforderung (Sirsch, 2000). Es werden die Hypothesen überprüft, wonach die Passungswahrnehmung, Lern- und Leistungszielorientierung abnehmen, die Leistungen und Noten in Deutsch und Mathematik vom 5. zum 7. Schuljahr weniger stark zunehmen. Dabei wird angenommen, dass die Abnahme beim Übertritt in die Sekundarstufe I besonders markant auftritt. Zudem wird die Hypothese getestet, dass die Veränderung der Passungswahrnehmung beim Übertritt in die Sekundarstufe I mit der Veränderung der Lern- und Leistungszielorientierung sowie der Leistungen und Noten in Deutsch und Mathematik zusammenhängt.

1 Der Übergangszeitpunkt wird zukünftig in den Kantonen Aargau und Basel-Landschaft am Ende des 6. Schuljahrs stattfinden. 


\section{$5 \quad$ Methode}

\subsection{Forschungsdesign}

Die Hypothesen werden unter Beizug von Daten aus dem Forschungsprojekt Wirkungen der Selektion ${ }^{2}$ (kurz: WiSel) bearbeitet. Das Projekt wurde in den Kantonen Aargau, Basel-Landschaft, Bern und Luzern durchgeführt. Die vier ausgewählten Kantone unterschieden sich im Übertrittszeitpunkt: In den Kantonen Aargau und Basel-Landschaft war der Übertritt in die Sekundarstufe I nach dem 5. Schuljahr, in den Kantonen Bern und Luzern nach dem 6. Schuljahr (Tabelle 1). Aufgrund dieses quasi-experimentellen Designs kann der Einfluss des Übertritts in die Sekundarstufe I auf die Passungswahrnehmung, die Lern- und Leistungszielorientierung sowie die Leistungen und Noten in Deutsch und Mathematik losgelöst vom Alter untersucht werden.

Tab. 1 Stichprobendesign und Stichprobengrössen

\begin{tabular}{|c|c|c|c|c|c|}
\hline & 5. Klasse & 6. Klasse & 7. Klasse & $N$ (weibl.) & $N$ (männl.) \\
\hline $\begin{array}{l}\text { Früher Übertritt } \\
\text { (Kt AG/BL) }\end{array}$ & Primar & Sek I & Sek I & 117 & 181 \\
\hline $\begin{array}{l}\text { Später Übertritt } \\
\text { (Kt BE/LU) }\end{array}$ & Primar & Primar & Sek I & 95 & 205 \\
\hline
\end{tabular}

Legende: Kt. AG/BL: Kantone Aargau/Basel-Landschaft, Kt. BE/LU: Kantone Bern/Luzern

\subsection{Stichprobe}

Im Jahr 2011 wurden insgesamt 99 Schulklassen mit 1802 Jugendlichen des 5. Schuljahres aus vier Kantonen zufällig ausgewählt. Die Kinder waren durchschnittlich 11-jährig. Diese Jugendlichen wurden im 6. und 7. Schuljahr erneut kontaktiert. Die Längsschnittstichprobe mit Daten zum 5., 6. und 7. Schuljahr umfasst 598 Jugendliche (Tabelle 1). Es wurden keine systematischen Rücklaufverzerrungen gefunden (Neuenschwander, Rösselet, Niederbacher, Rottermann \& Scheffler, 2014).

2 Ich danke dem Schweizerischen Nationalfonds für den finanziellen Beitrag an die Studie (Projektnummer 100013_13459471). Ich danke Dr. Benno Rottermann, Stephan Rösselet und Edith Niederbacher für die Arbeit bei der Durchführung der Studie. 


\subsection{Instrumente}

Es wurden standardisierte Schülerfragebogen und Leistungstests in Deutsch und Mathematik eingesetzt. Es folgt die Beschreibung der Skalen im Schülerfragebogen:

Das Geschlecht wurde mit der folgenden Frage erfasst: „Bitte gib dein Geschlecht an." Als Antwortkategorien dienten (1) weiblich und (2) männlich. Die Schichtzugehörigkeit (ISEI) wurde anhand der Berufe der ersten und der zweiten Bezugspersonen (Erziehungsberechtigte) zum ersten Messzeitpunkt ermittelt. Die Berufe wurden gemäß der International Standard Occupation Classification (ISCO08) codiert (International Labour Organization ILO, 2008). Anschließend wurde jedem Beruf anhand der ISCO-Codierung ein zweistelliger kontinuierlicher Wert basierend auf dem Standard International Socio-Economic Index of Occupational Status (ISEI-08) zugewiesen (Ganzeboom, 2010). Der jeweils höhere Wert der beiden Bezugspersonen wurde verwendet.

Die Passungswahrnehmung wurde mit acht Items operationalisiert, die auf einer 6-Punkte Skala mit den Polen 1 ,stimmt überhaupt nicht' und 6 , stimmt voll und ganz beantwortet werden konnten. Die Eingangsfrage lautete: „Wie sehr stimmen folgende Aussagen zum Unterricht in deiner Klasse für dich?" Die Items begannen mit: „Im Unterricht...“ Sie wurden fortgesetzt zum Beispiel mit „... bin ich sehr motiviert" oder „... erhalte ich interessante Aufgaben“ (Gesamte Stichprobe Welle 1: $\alpha=.82, M=4.84, S D=.65)$. Die Faktorenanalyse ergab einen Faktor, der $38.0 \%$ der Varianz aufklärte.

Die Lernzielorientierung als Indikator der Motivation wurde mit drei Items von Midgley, Maehr, Hruda et al. (2000) gemessen wie zum Beispiel „Mir ist es wichtig, die im Unterricht behandelten Themen vollständig zu verstehen“. Es wurde eine Ratingskala mit 6 Punkten ( 1 , trifft überhaupt nicht zu' und 6 , trifft voll und ganz $\mathrm{zu}$ ) verwendet $(M=5.162, S D=.67, \alpha=.62)$.

Die Leistungszielorientierung als Indikator der Motivation wurde mit drei Items von Midgley et al. (2000) gemessen wie zum Beispiel „Es ist mir wichtig, im Vergleich mit den anderen in meiner Klasse klug zu wirken". Es wurde eine Ratingskala mit 6 Punkten ( 1 , trifft überhaupt nicht zu' bis 6 , trifft voll und ganz zu') verwendet ( $M$ $=3.62, S D=1.19, \alpha=.80$ ).

Die Noten in Deutsch und Mathematik wurden mit der folgenden Frage erfasst: „Wie sind deine Noten? Unten steht eine Liste mit verschiedenen Fächern. Gib bitte für diejenigen Fächer, die du im letzten Jahr besucht hast, deine letzten Zeugnisnoten an. Kreuze bei allen anderen Fächern bitte „keine Note“ an." Es folgte eine Liste mit Schulfächern. Die Noten wurden nach der Schweizer Notenskala erfasst mit den Extremen 1 (sehr schlecht)und 6 (sehr gut), wobei Noten unterhalb von 4 als 
ungenügend bewertet werden. Die Noten wurden zu allen drei Messzeitpunkten rückblickend auf das vergangene Schuljahr erfragt.

Der Mathematiktest der ersten Welle enthielt sieben Konzepte mit insgesamt 39 Items, die dichotom kodiert wurden (falsch: 0 Punkte, richtig: 1 Punkt). Die Split-half-Reliabilitäten erste Testhälfte vs. zweite Testhälfte war $r_{\mathrm{SH}}=.80$, gerade vs. ungerade Items $r_{\mathrm{SH}}=.87$ (Berechnung mit Gesamtstichprobe zu $\mathrm{t}_{1}$ ). Der Mathematiktest der zweiten Welle umfasste zehn Konzepte mit 45 Items. Die Split-half-Reliabilitäten erste Hälfte gegen zweite Hälfte $r_{\mathrm{SH}}=.82$, gerade vs. ungerade Items $r_{\mathrm{SH}}=.91$, Gesamtstichprobe $t_{2}$ waren gut. Der Mathematiktest der dritten Welle bestand aus acht Konzepten mit 55 Items. Die Split-half-Reliabilitäten erste Hälfte gegen zweite Hälfte $r_{\mathrm{SH}}=.77$, gerade vs. ungerade Items $r_{\mathrm{SH}}=.91$, Gesamtstichprobe $\mathrm{t}_{3}$ waren gut (Neuenschwander, Rottermann, Niederbacher, Rösselet \& Scheffler, 2014).

Der Deutschtest der ersten Welle umfasste sechs Konzepte mit insgesamt 83 Items. Analog zum Mathematiktest wurden die Items ebenfalls dichotom mit 0 und 1 kodiert. Die Split-half-Reliabilitäten erste Testhälfte vs. zweite Testhälfte $\underline{\underline{r}}_{\underline{H}}=.80$ und gerade vs. ungerade Items $r_{\mathrm{SH}}=.92$, aus der Gesamtstichprobe Welle 1 waren gut. Der in der zweiten Welle eingesetzte Deutschtest enthielt zehn Konzepte mit insgesamt 85 Items. Die Split-half-Reliabilitäten erste Hälfte gegen zweite Hälfte $r_{\mathrm{SH}}=.82$, gerade vs. ungerade Items $r_{\mathrm{SH}}=.95$, der Gesamtstichprobe $\mathrm{t}_{2}$ waren gut. Der Deutschtest der dritten Welle bestand aus elf Aufgaben mit insgesamt 97 Items. Split-half-Reliabilitäten erste Hälfte gegen zweite Hälfte $r_{\mathrm{SH}}=.85$, gerade vs. ungerade Items $r_{\mathrm{SH}}=.95$, der Gesamtstichprobe $\mathrm{t}_{3}$ waren gut.

In den Tests beider Fächer wurde in einem Ankeritem-Design eine Teilmenge der Items des früheren Messzeitpunkts zum jeweils folgenden Messzeitpunkt vorgelegt. Basierend auf der Item Response-Theorie (IRT; Yen \& Fitzpatrick, 2006) wurde mit der Software R die Linking-Methode von Haberman (2009) angewendet, mit welcher die Schülerleistungen auf einer Metrik abgebildet werden, wodurch die tatsächliche Veränderung der Kompetenzwerte über die drei Messzeitpunkte beschrieben werden kann. Als Kompetenzwerte der Schülerinnen und Schüler wurden sogenannte Weighted-likelihood-Werte (Warm, 1989) ermittelt (Mittelwerte: $t_{1}$ : Mathematik $M=.03, S D=1.09, N=1499$, Deutsch $M=.04, S D=1.04, N=1491 ; \mathrm{t}_{2}$ : Mathematik $M=.51$, $S D=.99, N=1820$, Deutsch $M=.25, S D=1.13, N=1818$; $\mathrm{t}_{3}$ : Mathematik: $M=.60, S D=.96$, $N=1499$, Deutsch $M=.52, S D=1.18, N=1493)$. 


\section{$6 \quad$ Ergebnisse}

\subsection{Veränderung der Passungswahrnehmung}

Um die Hypothese zu prüfen, dass die Passungswahrnehmung mit einem frühen Übertritt von der Primarschule in die Sekundarstufe I stärker sinkt als bei Gleichaltrigen, die später in die Sekundarstufe I übertreten, wurden Kovarianzanalysen mit Messwiederholung gerechnet. Es wurde der Verlauf der Passungswahrnehmung vom 5. zum 7. Schuljahr zwischen der Gruppe mit einem Übertritt in die Sekundarstufe I nach dem 6. Schuljahr (später Übertritt) und der Gruppe mit einem Übertritt in die Sekundarstufe I nach dem 5. Schuljahr (früher Übertritt) verglichen. Ein signifikanter Interaktionseffekt zeigt, dass sich die Verläufe zwischen den zwei Gruppen unterscheiden. Ein linearer Interaktionseffekt zeigt, dass sich die Mittelwertverläufe je nach Übertrittszeitpunkt unterscheiden. Ein quadratischer Interaktionseffekt indiziert, dass sich die Mittelwerte vor bzw. nach dem Übertritt stärker bzw. schwächer als während des Übertritts und im Vergleich zur anderen Gruppe verändern. D.h. , der Übertritt führt zu einer spezifischen $\mathrm{Zu}$ - oder Abnahme eines Merkmals. Es werden die linearen und die quadratischen Effekte ausgewiesen, um die Art der Veränderung des jeweiligen Merkmals präziser beschreiben zu können.

Die beiden Gruppen können sich aufgrund kantonsspezifischer Merkmale unterscheiden, so dass Effekte nicht eindeutig als Effekte des Übertrittszeitpunkts interpretiert werden können. Daher werden die Daten der Jugendlichen aus je zwei Kantonen, in denen die Jugendlichen zum gleichen Zeitpunkt übertreten, zusammengefasst ausgewertet. Überdies werden Kovariaten einbezogen, welche einen Einfluss auf die abhängige Variable haben können und in denen sich die beiden Gruppen unterscheiden können. Konkret werden die Kovariaten Geschlecht, Schicht der Eltern, Nationalität des Kindes sowie die vom Jugendlichen eingeschätzte Wichtigkeit der Schule kontrolliert. Mit diesen Kovariaten wird ein allfälliger Störeffekt von dritten Variablen auf diese Verläufe kontrolliert.

Tab. 2 Veränderung der Passungswahrnehmung: Geschätzte Mittelwerte mit Standardfehlern

\begin{tabular}{lcc}
\hline & früher Übertritt $(N=171)$ & später Übertritt $(N=293)$ \\
\hline 5. Klasse & $4.82(.04)$ & $4.84(.03)$ \\
6. Klasse & $4.73(.05)$ & $4.80(.04)$ \\
7. Klasse & $4.56(.05)$ & $4.72(.04)$ \\
\hline
\end{tabular}

Kovariaten: Geschlecht, Schicht der Eltern, Nationalität des Kindes, vom Jugendlichen eingeschätzte Wichtigkeit der Schule 
Der Haupteffekt Übertrittszeitpunkt war nicht signifikant ( $F$ übertrittszeitpunkt $(1$, $458)=3.1)$, der lineare Zeiteffekt war signifikant $\left(F_{\text {linear Zeit }}(1,458)=21.5, p<.001, F\right.$ quadr Zeit $(1,458)=2.4, n s)$, der lineare Interaktionseffekt war auch signifikant $\left(F_{\text {linear }}\right.$ Interaktion $\left.(1,458)=5.0, p<.05, F_{\text {quadr Interaktion }}(1,458)=0.09, n s\right)$. Die Mittelwerte in Tabelle 2 zeigen in beiden Gruppen ein deutlicheres Sinken der Passungswahrnehmung beim Übertritt und nach Eintritt in die Sekundarstufe I als in der Primarschule. Während die Passungswahrnehmung bei der Gruppe mit spätem Übertritt vom 5. zum 6. Schuljahr in der Primarschule wenig abnimmt, nimmt sie bei den gleichaltrigen Jugendlichen in der Gruppe mit frühem Übertritt stärker ab. Auch vom 6. zum 7. Schuljahr ist die Abnahme in der Gruppe später Übertritt, wenn die Kinder den Übertritt in die Sekundarstufe I vollziehen, kleiner als in der Gruppe der Gleichaltrigen mit frühem Übertritt, welche sich in der Sekundarstufe I befinden. Weil die Jugendlichen mit dem Übertritt in die Sekundarstufe I in unterschiedliche Schulniveaus übertreten, wurde in Kontrollanalysen ein Effekt des Schulniveaus überprüft. Diese Analysen zeigen für die Passungswahrnehmung keinen signifikanten Effekt. Die Ergebnisse stützen die formulierte Hypothese, dass die Passungswahrnehmung in der Gruppe mit frühem Übertritt in die Sekundarstufe I stärker sinkt als in der Gruppe mit spätem Übertritt.

\subsection{Veränderung der Lern- und Leistungszielorientierung}

Im nächsten Schritt wird die Hypothese überprüft, ob analog zur Passungswahrnehmung die Lernziel- und die Leistungszielorientierung in der Gruppe mit frühem Übertritt stärker sinken als in der Gruppe mit spätem Übertritt. Es wurden einfaktorielle Kovarianzanalysen mit Messwiederholung gerechnet. Als Kovariaten wurden Geschlecht, Schicht der Eltern, Nationalität des Kindes sowie die vom Jugendlichen eingeschätzte Wichtigkeit der Schule verwendet. Bei der Lernzielorientierung war der Haupteffekt Übertrittszeitpunkt nicht signifikant $\left(F_{\text {Übertrittszeitpunkt }}(1,451)=1.5, n s\right)$, der lineare und der quadratische Zeiteffekt waren signifikant $\left(F_{\text {linear Zeit }}(1,451)=8.3, p<.01 ; F_{\text {quadr Zeit }}(1,451)=6.5, p<.05\right)$ und der lineare Interaktionseffekt war auch signifikant $\left(F_{\text {linear Interaktion }}(1,451)=7.1, p<.01\right.$; $\left.F_{\text {quadr Interaktion }}(1,451)=.02, n s\right)$. Gemäss der Mittelwerte in Tabelle 3 nimmt analog zur Passungswahrnehmung die Lernzielorientierung in der Gruppe mit frühem Übertritt stärker ab als in der Gruppe mit spätem Übertritt. 
Tab. 3 Veränderung der Leistungen in Deutsch und Mathematik (Mittelwerte und Standardfehler)

\begin{tabular}{lcccc}
\hline & \multicolumn{2}{c}{$\begin{array}{c}\text { Lernzielorientierung } \\
\text { früher Übertritt } \\
N=169\end{array}$} & $\begin{array}{c}\text { später Übertritt } \\
N=288\end{array}$ & $\begin{array}{c}\text { Leistungszielorientierung } \\
\text { früher Übertritt } \\
N=169\end{array}$ \\
$\begin{array}{lcccc}\text { später Übertritt } \\
N=286\end{array}$ \\
\hline 5. Klasse & $5.14(.04)$ & $5.10(.03)$ & $3.51(.09)$ & $3.41(.07)$ \\
6. Klasse & $5.02(.05)$ & $5.09(.04)$ & $3.25(.10)$ & $3.31(.07)$ \\
7. Klasse & $4.78(.05)$ & $4.94(.04)$ & $3.23(.10)$ & $2.98(.07)$ \\
\hline
\end{tabular}

Kovariaten: Geschlecht, Schicht der Eltern, Nationalität des Kindes, vom Jugendlichen eingeschätzte Wichtigkeit der Schule

Bei der Leistungszielorientierung war der Haupteffekt Übertrittszeitpunkt nicht signifikant $\left(F_{\text {Úbertrittszeitpunkt }}(1,447)=1.0, n s\right)$, der lineare und quadratische Zeiteffekt war nicht signifikant $\left(F_{\text {linear Zeit }}(1,447)=.1, n s ; F_{\text {quadr Zeit }}(1,447)=.6\right.$, ns), der lineare Interaktionseffekt war nicht signifikant $\left(F_{\text {linear Interaktion }}(1,447)=1.4 n s\right)$, aber der quadratische Interaktionseffekt war signifikant $\left(F_{\text {quadratisch Interaktion }}(1,447)=5.6\right.$, $p<.05)$. Kontrollanalysen zeigen, dass die Ergebnisse dieser Analysen unabhängig vom Schulniveau sind, das die Schülerinnen und Schüler in der Sekundarstufe I besuchen. Die Mittelwerte in Tabelle 3 zeigen, dass die Leistungszielorientierung in der Gruppe mit frühem Übertritt vor allem vom 5. zum 6. Schuljahr, das heisst beim Übertritt in die Sekundarstufe I, abnimmt und dass sie in der Gruppe später Übertritt vor allem vom 6. zum 7. Schuljahr abnimmt, d. h. beim Übertritt von der Primarschule in die Sekundarstufe I. Die Abnahme der Leistungszielorientierung trat also bei gleichem Alter der Schülerinnen und Schüler vor allem beim Übertritt von der Primarschule in die Sekundarstufe I auf. Analog zur Passungswahrnehmung nahmen auch die Lern- und Leistungszielorientierung besonders beim Übertritt in die Sekundarstufe I ab.

\subsection{Veränderung der Leistungen und der Noten}

Im nächsten Schritt wurde überprüft, ob sich analog zur Passungswahrnehmung die Leistungen in Deutsch und Mathematik veränderten. Wiederum wurden Kovarianzanalysen mit Messwiederholung gerechnet. Als Kovariaten wurden Geschlecht, Schicht der Eltern, Nationalität des Kindes sowie die vom Jugendlichen eingeschätzte Wichtigkeit der Schule verwendet. Für die Leistungen in Deutsch war der Haupteffekt nicht signifikant $\left(F_{\text {Ubbertrittszeitpunkt }}(1,436)=.0, n s\right)$, der lineare Zeiteffekt war nicht signifikant $\left(F_{\text {linear Zeit }}(1,436)=3.3, n s ; F_{\text {quadr Zeit }}(1,436)=.2, n s\right)$, der lineare 
Interaktionseffekt war signifikant $\left(F_{\text {linear Interaktion }}(1,446)=9.0, p<.01 ; F_{\text {quadr Interaktion }}(1\right.$, 446)=1.8, ns). Gemäss Tabelle 4 nahmen die Testleistungen Deutsch in der Gruppe mit spätem Übertritt deutlich stärker zu als in der Gruppe mit frühem Übertritt.

Tab. 4 Veränderung der Leistungen in Deutsch und Mathematik (Mittelwerte und Standardfehler)

\begin{tabular}{|c|c|c|c|c|}
\hline & \multicolumn{2}{|c|}{ Deutsch } & \multicolumn{2}{|c|}{ Mathematik } \\
\hline & $\begin{array}{c}\text { früher Übertritt } \\
\qquad N=158\end{array}$ & $\begin{array}{c}\text { später Übertritt } \\
\qquad N=285\end{array}$ & $\begin{array}{c}\text { früher Übertritt } \\
\qquad N=160\end{array}$ & $\begin{array}{c}\text { später Übertritt } \\
N=286\end{array}$ \\
\hline 5. Kl. & $.31(.07)$ & $.15(.06)$ & $.30(.08)$ & $-.01(.06)$ \\
\hline 6. Kl. & $.31(.08)$ & $.38(.06)$ & $.53(.07)$ & $.56(.05)$ \\
\hline 7. Kl. & $.59(.09)$ & $.70(.06)$ & $.58(.08)$ & $.68(.06)$ \\
\hline
\end{tabular}

Kovariaten: Geschlecht, Schicht der Eltern, Nationalität des Kindes, vom Jugendlichen eingeschätzte Wichtigkeit der Schule

Für die Leistungen in Mathematik war der Haupteffekt nicht signifikant ( $F$ übertrittszeitpunkt $(1,439)=.5, n s)$, der lineare Zeiteffekt war signifikant $\left(F_{\text {linear Zeit }}(1,439)=5.4\right.$, $\left.p<.05 ; F_{\text {quadr Zeit }}(1,439)=.6, n s\right)$ und der lineare und quadratische Interaktionseffekt war auch signifikant $\left(F_{\text {linear Interaktion }}(1,439)=20.3, p<.001 ; F_{\text {quadr Interaktion }}(1,439)=4.8\right.$, $p<.05)$. Kontrollanalysen zeigen allerdings, dass die Veränderung der Leistungen in Deutsch und Mathematik vom Schulniveau, das die Schülerinnen und Schüler in der Sekundarstufe I besuchen, abhängt. Die Leistungszunahme ist beim Übertritt in ein Schulniveau der Sekundarstufe I mit hohen Anforderungen grösser als beim Übertritt in ein Schulniveau mit Grundanforderungen. Auch nach Kontrolle des Schulniveaus nehmen die Leistungen in Deutsch und Mathematik in der Gruppe mit spätem Übertritt deutlich stärker zu als in der Gruppe mit frühem Übertritt. Die Leistungszunahme in der Primarschule war grösser als beim Übertritt in die Sekundarstufe I und die Leistungszunahme beim Übertritt in die Sekundarstufe I war grösser als beim Verbleib in der Sekundarstufe I. Die Ergebnisse zeigen, dass die Leistungen für Deutsch und Mathematik im 7. Schuljahr bei den Jugendlichen mit spätem Übertritt höher sind als bei den Jugendlichen mit frühem Übertritt. Ein später Übertritt in die Sekundarstufe I begünstigt in Übereinstimmung mit den Hypothesen höhere Leistungen in Deutsch und Mathematik.

Um die Veränderung der Noten in Deutsch und Mathematik beim Übertritt in die Sekundarstufe zu beschreiben, wurden Kovarianzanalysen mit Messwiederholung gerechnet. Als Kovariaten wurden wiederum Geschlecht, Schicht der Eltern, Nationalität des Kindes sowie die vom Jugendlichen eingeschätzte Wichtigkeit 
der Schule verwendet. Die Noten liegen für das 4., 5. und 6. Schuljahr vor. Gemäss Tabelle 5 weisen die Schülerinnen und Schüler mit spätem Übertritt bessere Noten in Deutsch als die anderen Schülerinnen und Schüler auf ( $F$ Übertrittszeitpunkt (1, $435)=16.0, p<.001)$. Der Zeiteffekt ist nicht signifikant $\left(F_{\text {linear Zeit }}(1,435)=1.8\right.$, ns; $F$ quadr Zeit $(1,435)=.2, n s)$, die Interaktion Übertrittszeitpunkt mal Zeit ist signifikant $(F$ linear Interaktion $\left.(1,435)=13.2, p<.001 ; F_{\text {quadr Interaktion }}(1,435)=28.3, p<.001\right)$. Auch bei den Noten in Mathematik war der Übertrittszeitpunkteffekt signifikant ( $F_{\text {Ubertrittszeitpunkt }}$ $(1,437)=11.0, p<.001)$, der Zeiteffekt zeigte keine Abnahme der Noten in Mathematik $\left(F_{\text {linear Zeit }}(1,437)=.33, n s ; F_{\text {quadr Zeit }}(1,437)=1.0, n s\right)$. Die Interaktion belegte hypothesenkonform eine stärkere Abnahme der Noten nach dem Übertritt in die Sekundarstufe I in der 5. Klasse zur 6. Klasse als bei den Gleichaltrigen mit Verbleib in der Primarstufe $\left(F_{\text {linear Interaktion }}(1,437)=6.9, p<.01 ; F_{\text {quadr Interaktion }}(1,437)=27.9\right.$, $p<.001)$. Wird das besuchte Schulniveau in der Sekundarstufe I als zusätzlicher Faktor einbezogen, verändern sich diese Effekte bezüglich der Noten in Deutsch und Mathematik nicht. Die Interaktion Übertrittszeitpunkt mal Schulniveau mal Zeit wird in beiden Fächern signifikant. Beim Übertritt in ein Schulniveau mit tiefen Anforderungen nehmen die Noten zu, während sie beim Übertritt in ein Schulniveau mit höheren Anforderungen abnehmen. In der Gruppe mit frühem Übertritt zeigt sich dieser Effekt vom 5. zum 7. Schuljahr, während er bei der Gruppe mit spätem Übertritt nur vom 6. zum 7. Schuljahr auftritt.

Tab. 5 Veränderung der Noten in Deutsch und Mathematik (Mittelwerte und Standardfehler)

\begin{tabular}{lcccc}
\hline & \multicolumn{2}{c}{ Deutsch } & \multicolumn{2}{c}{ Mathematik } \\
& $\begin{array}{c}\text { früher Übertritt } \\
N=160\end{array}$ & $\begin{array}{c}\text { später Übertritt } \\
N=281\end{array}$ & $\begin{array}{c}\text { früher Übertritt } \\
N=161\end{array}$ & $\begin{array}{c}\text { später Übertritt } \\
N=282\end{array}$ \\
\hline $4 . \mathrm{Kl}$. & 4.99 & 5.12 & 4.95 & 5.11 \\
$5 . \mathrm{Kl}$. & 4.99 & 5.03 & 4.93 & 4.97 \\
$6 . \mathrm{K} 1$. & 4.72 & 5.04 & 4.59 & 4.90 \\
\hline
\end{tabular}

Kovariaten: Geschlecht, die Schicht der Eltern, die Nationalität des Kindes sowie die vom Jugendlichen eingeschätzte Wichtigkeit der Schule

\subsection{Korrelationen}

Um den Zusammenhang zwischen der Passungswahrnehmung und den Leistungsindikatoren zu testen, wurden Korrelationsanalysen gerechnet. Die Passungswahrnehmung korrelierte wie erwartet mit den Leistungen in Mathematik ( $r=.10$, 
$p<.05)$, mit den Noten im 5. Schuljahr in Deutsch $(r=.12, p<.01)$ und Mathematik $(r=.20, \mathrm{p}<.001)$ sowie mit der Lern- $(r=.15, p<.001)$ und Leistungszielorientierung $(r=.54, p<.001)$ signifikant. Einzig die Korrelation der Passungswahrnehmung mit den Leistungen in Deutsch war nicht signifikant $(r=.04, n s)$. Die Passungswahrnehmung hängt also erwartungsgemäss mit Indikatoren der Motivation und Leistungen zusammen.

Im nächsten Schritt wurde überprüft, ob das Ausmass der Veränderung der Passungswahrnehmung vom 5. zum 6. Schuljahr mit dem Ausmass der Veränderung der Leistungen in Mathematik und Deutsch, in den Noten in Mathematik und Deutsch und in den Motivationsindikatoren Leistungszielorientierung und Lernzielorientierung zusammenhingen. Wenn die präsentierten Veränderungen der Leistungen, Noten und Motivation beim Übertritt in die Sekundarstufe I durch die Passungswahrnehmung erklärt werden könnten, müsste es systematische Kovariationen in der Differenz der Passungswahrnehmung vom 5. zum 6. Schuljahr mit der Differenz der genannten Leistungsindikatoren vom 5. zum 6. Schuljahr geben. Weil die Noten im 7. Schuljahr nicht vorliegen, wurde diese Analyse nur für die Veränderung vom 5. zum 6. Schuljahr gerechnet, aber getrennt für die Gruppen mit Übertritt und ohne Übertritt. Die Ergebnisse sind in Tabelle 6 dargestellt.

Tab. 6 Pearson-Korrelationen: Veränderung der Passungswahrnehmung und Veränderung der Leistungen und Noten in Deutsch und Mathematik und der Leistungs- und Lernzielorientierung vom 5. zum 6. Schuljahr in den Gruppen früher Übertritt und später Übertritt.

\begin{tabular}{lcccccc}
\hline Passung & $\begin{array}{c}\text { Leistung } \\
\mathrm{M}\end{array}$ & $\begin{array}{c}\text { Leistung } \\
\mathrm{D}\end{array}$ & $\begin{array}{c}\text { Note } \\
\mathrm{M}\end{array}$ & $\begin{array}{c}\text { Note } \\
\mathrm{D}\end{array}$ & $\begin{array}{c}\text { Leistungs- } \\
\text { ziel }\end{array}$ & $\begin{array}{c}\text { Lernziel } \\
\text { Übertritt }\end{array}$ \\
$\begin{array}{l}(184<N<198) \\
\text { Kein Übertritt }\end{array}$ & .13 & -.12 & $.21^{* *}$ & $.20^{* *}$ & $.19^{* *}$ & $.39^{* * *}$ \\
$(322<N<330)$ & .10 & .04 & -.08 & .10 & .07 & $.28^{* * *}$ \\
\hline
\end{tabular}

Legende: ${ }^{* * *}: p<.001,{ }^{* *}: p<.01,{ }^{*}: p<.05$

Beim Übertritt von der Primarschule in die Sekundarstufe I gibt es hypothesenkonform signifikante positive Korrelationen zwischen der Veränderung der Passungswahrnehmung und der Veränderung der Noten in Mathematik und Deutsch, aber auch der Leistungszielorientierung und Lernzielorientierung. Es gibt aber keine signifikanten Korrelationen mit der Veränderung der Leistungen im Mathematikund Deutschtest. In der Gruppe ohne Übertritt vom 5. zum 6. Schuljahr findet sich nur eine signifikante Korrelation (Veränderung der Passungswahrnehmung 
mit Veränderung der Lernzielorientierung). Möglicherweise ist die Varianz der Differenzwerte in der Gruppe ohne Übertritt zu klein, weil sich die Leistungen, Noten und Lern- und Leistungszielorientierung vom 5. zum 6. Schuljahr kaum verändern (vgl. Tabellen 2-5).

Diese deskriptiven Analysen zeigen, dass die Veränderungen der Noten und der Lern- und Leistungszielorientierung beim Übertritt in die Sekundarstufe mit der Veränderung der Passungswahrnehmung systematisch kovariieren. Mit dem Absinken der Passungswahrnehmung in die Sekundarstufe I sinken auch die Lernund Leistungszielorientierung und der Noten.

\section{Schlussfolgerungen}

Die Ergebnisse zeigen in Übereinstimmung mit Eccles (2004), dass analog zur USA auch in der Schweiz die Passungswahrnehmung beim frühen Übertritt in die Sekundarstufe I bei gleichem Alter der Kinder stärker abnimmt als bei einem späten Übertritt. Damit korrespondieren die Befunde, dass beim Übertritt in die Sekundarstufe I auch die Lern- und die Leistungszielorientierung deutlich stärker abnehmen und die Leistungen in Deutsch und Mathematik weniger zunehmen. Das ist nicht primär ein Alterseffekt, sondern ein Effekt der institutionellen Strukturierung und der Lehrpraxis im Unterricht. Die geringere Leistungszunahme in der Sekundarstufe I im Vergleich zur Primarschule berichteten auch Angelone, Keller \& Moser (2013) sowie Moser, Oostlander, Tomasik (in diesem Band). Sie erklärten diese mit der unterschiedlichen Struktur der Lehrpläne in der Primarschule im Vergleich zur Sekundarstufe I.

Diese deskriptiven Ergebnisse lassen keine kausalen Erklärungen zu, wonach die Motivationsabnahme bzw. die geringere Leistungszunahme auf die geringere Passung zwischen individuellen Interessen und Wissen und den Unterrichtsangeboten zurückzuführen ist. Dennoch gibt es beim Übertritt in die Sekundarstufe I zwischen den Veränderungen der Passungswahrnehmung und der Leistungsindikatoren systematische Zusammenhänge. Dies stützt die Interpretation, dass die Veränderung der Noten und der Lern- und Leistungszielorientierung mit der Abnahme der Passungswahrnehmung beim Übertritt in die Sekundarstufe I erklärt werden kann.

Die Gründe für die Abnahme der Passungswahrnehmung sind unzureichend untersucht. Ausgehend von Eccles (2004) Analyse zu US-amerikanischen Schulen wird vermutet, dass in der Sekundarstufe I der Unterricht weniger entwicklungsangepasst ist als in der Primarstufe. Der Unterricht ist fachorientiert und berücksichtigt die individuellen Interessens- und Wissensvoraussetzungen weniger stark 
als in der Primarschule. Die häufigste Unterrichtsform auf der Sekundarstufe I ist der lehrerzentrierte Unterricht (Ludwig-Tauber et al., 2000), der vermutlich die individuellen Interessens- und Vorwissenslagen der Schülerinnen und Schüler unzureichend aufnimmt. Möglicherweise ist der Unterricht in der Sekundarstufe I weniger adaptiv an den Interessen und am Wissen der Kinder ausgerichtet als in der Primarschule. Es könnte sein, dass es in der Sekundarstufe I eine ausgewogenere Balance zwischen den fachlichen und pädagogischen Zielen der Lehrperson und den Bedürfnissen der einzelnen Schülerinnen und Schüler braucht.

Interessant ist, dass sich die Gruppierung der Kinder in leistungshomogene Schulklassen aufgrund der Einteilung in Schulniveaus nicht in einer höheren Passungswahrnehmung, Motivation und Leistungszunahme auswirkt. Die Hoffnung, die Lernbedingungen durch eine Leistungsgruppierung in Schulniveaus zu verbessern, wird innerhalb von 1.5 Jahren nach Eintritt in die Sekundarstufe I nicht erfüllt.

Einschränkend muss angemerkt werden, dass die formulierten Erklärungen zur Veränderung von Passung, Motivation und Leistungsindikatoren beim Übertritt in die Sekundarstufe I nicht spezifisch überprüft werden konnten. Die Ergebnisse belegen je nach Übertrittszeitpunkt unterschiedliche Verläufe, über deren Erklärung aber nur spekuliert werden kann. Die Erklärung dieser Übergangsmuster sollte in zukünftigen Studien überprüft werden. Die Befunde werden gleichwohl als wichtig erachtet, weil sie neu sind und das Verständnis des Übertritts in die Sekundarstufe I vertiefen.

\section{Literatur}

Alspaugh, J. W., \& Harting, R. D. (1995). Transition effects of school grade-level organization on student achievement. Journal of Research and Development in Education, 28, 145-149.

Alspaugh, J. W. (1998). Achievement loss associated with the transition to middle school and high school. Journal of Educational Research, 92, 20-26.

Angelone, D., Keller, F., \& Moser, U. (2013). Entwicklung schulischer Leistungen während der obligatorischen Schulzeit. Bericht zur vierten Zürcher Lernstandserhebung zuhanden der Bildungsdirektion des Kantons Zürich. Zürich: Institut für Bildungsevaluation.

Conley, A. M. (2012). Patterns of motivation beliefs: Combining achievement goal and expectancy-value perspectives. Journal of Educational Psychology, 104(1), 32-47. doi:10.1037/a0026042

Eccles, J. S., \& Roeser, R. W. (2009). Schools, academic motivation, and stage-environment fit. In R. M. Lerner \& L. Steinberg (Eds.), Handbook of adolescent psychology (3rd ed., pp. 404-434). Hoboken, N.J: John Wiley \& Sons. 
Eccles, J. S. (2004). Schools, academic motivation, and stage-environment fit. In R. M. Lerner \& L. Steinberg (Eds.), Handbook of adolescent psychology (2nd ed., Vol. 2, pp. 125-153). Hoboken, NJ: Wiley.

Eccles, J. S., Midgley, C., Wigfield, A., Buchanan, C. M., Reuman, D., Flanagan, C., \& Mac Iver, D. (1993). Development during adolescence: the impact of stage-environment fit on young adolescents' experiences in schools and in families. American Psychologist, 48(2), 90-101.

Ganzeboom, H. B. G., \& Treiman, D. J. (2010). International stratification and mobility file: Conversion Tools. Amsterdam: Department of Social Research Methodology.

Götz, T., Lohrmann, K., Ganser, B., \& Haag, L. (2005). Einsatz von Unterrichtsmethoden Konstanz oder Wandel? Empirische Pädagogik, 19(4), 342-360.

Haberman, S. J. (2009). Linking parameter estimates derived from an item response model through separate calibrations. ETS Research Report No. RR-09-40. Princeton, NJ: ETS.

Kristof-Brown, A. L., Zimmermann, R. D., \& Johnson, E. C. (2005). Consequences of individual's fit at work: A meta-analysis of person-job, person-organization, person-group, and person-supervisor fit. Personnel Psychology, 58, 1-342.

Ludwig-Tauber, M., Wild-Naef, M., \& Vouets, V. (2000). Merkmale des Berufsfeldes von Lehrerinnen und Lehrern der 7. bis 9. Klasse. Eine Studie im Auftrag der Erziehungsdirektion des Kantons Bern. Bern: Forschungsstelle für Schulpädagogik und Fachdidaktik, Universität Bern.

Midgley, C., Maehr, M., Hruda, L., Anderman, E., Anderman, L., Freeman, K., Gheen, M., Kaplan, A., Kumar, R., Middleton, M.J., Nelson, J., Roeser, R., \& Urdan, T. (2000). Manual for the patterns of adaptive learning scales. Ann Arbor, MI: University of Michigan.

Maaz, K., Hausen, C., McElvany, N., \& Baumert, J. (2006). Stichwort: Übergänge im Bildungssystem - Theoretische Konzepte und ihre Anwendung in der empirischen Forschung beim Übergang in die Sekundarschule. Zeitschrift für Erziehungswissenschaft, 9(3), 299-327.

Moser, U., Oostlander, J., \& Tomasik, M. J. (2017). Soziale Ungleichheiten im Leistungszuwachs und bei Bildungsübergängen. In M. P. Neuenschwander \& C. Nägele (Hrsg.), Bildungsverläufe von der Einschulung in die Erwerbstätigkeit: Theoretische Ansätze - Befunde - Beispiele (S. 59-77). Wiesbaden: Springer VS.

Neuenschwander, M. P. \& Malti, T. (2009). Selektionsprozesse beim Übergang in die Sekundarstufe I und II. Zeitschrift für Erziehungswissenschaft, 12(2), 216-232.

Neuenschwander, M. P. (2016). Bildungsungleichheit am Beispiel der Leistungsentwicklung in Deutsch und Mathematik beim Übertritt in die Sekundarstufe I. In B. Ziegler (Hrsg.), (Un-)Gleichheiten in der Demokratie (S. 95-118). Zürich: Schulthess-Verlag.

Neuenschwander, M. P. (2017). Lern- und Leistungszielorientierung beim Übergang in die Sekundarstufe I: Längsschnittliche Befunde zur Bedeutung von Belastungen und Erziehungsverhalten von Eltern. Schweizerische Zeitschrift für Bildungswissenschaften, 39(2).

Neuenschwander, M. P., Rottermann, B., Niederbacher, E., Rösselet, S., \& Scheffler, L. (2014). Wirkungen der Selektion WiSel. Dokumentation der Leistungstests Mathematik und Deutsch der Welle 3 2013/2014. Solothurn: Zentrum Lernen und Sozialisation der PH FHNW.

Neuenschwander, M. P., Rösselet, S., Niederbacher, E., Rottermann, B., \& Scheffler, L. (2014). Wirkungen der Selektion WiSel. Dokumentation des Fragebogens für Schülerinnen und Schüler der Welle 3 2013/2014. Solothurn: Zentrum Lernen und Sozialisation der PH FHNW.

Pauli, C., Reusser, K., Waldis, M., \& Grob, U. (2003). „Erweiterte Lehr- und Lernformen“ im Mathematikunterricht der Deutschschweiz. Unterrichtswissenschaft, 31(4), 291-320.

Piaget, J. (1947). La psychologie de l'intelligence (dt. Psychologie der Intelligenz. Stuttgart: Klett, Trans. 8 ed.). Paris: Colin. 
Pintrich, P. R., Conley, A. M., \& Kempler, T. M. (2003). Current issues in achievement goal theory and research. International Journal of Educational Research, 39, 319-337. doi:10.1016/j. ijer.2004.06.002

Reusser, K., Pauli, C., \& Waldis, M. (Hrsg.). (2010). Unterrichtsgestaltung und Unterrichtsqualität. Münster: Waxmann.

Rösselet, S., \& Neuenschwander, M. P. (2017). Akzeptanz und Ablehnung von Schülerinnen und Schülern beim Übertritt in die Sekundarstufe I. In M. P. Neuenschwander \& C. Nägele (Eds.), Bildungsverläufe von der Einschulung in die Erwerbstätigkeit: Theoretische Ansät$z e-$ Befunde - Beispiele (pp. 103-121). Wiesbaden: VS Verlag für Sozialwissenschaften.

Sirsch, U. (2000). Probleme beim Schulwechsel. Münster: Waxmann.

Sternberg, R. J. (1997). The concept of intelligence and its role in lifelong learning and success. American Psychologist, 52(2), 1030-1038.

Van Ophuysen, S. (2008). Zur Veränderung der Schulfreude von Klasse 4- bis 7. Eine Längsschnittanalyse schulformspezifischer Effekte von Ferien und Grundschulübergang. Zeitschrift für Pädagogische Psychologie, 22, 293-306.

Whitley, J., Lupart, J. L. \& Beran, T. (2007). Differences in achievement between adolescents who remain in a K-8 school and those who transition to a junior high school. Canadian Journal of Education/Revue canadienne de l'éducation, 649-669.

Yen, W. M. \& Fitzpatrick, A. R. (2006). Item response theory. In R. L. Brennan (Ed.), Educational Measurement (pp. 111-154). Westport: Praeger Publisher.

Zimmer-Gembeck, M. J., Chipuer, H. M., Hanisch, M., Creed, P. A. \& McGregor, L. (2006). Relationships at school and stage-environment fit as resources for adolescent engagement and achievement. Journal of Adolescent Health, 29(6), 911-933. doi:10.1016/j.adolescence.2006.04.008

Open Access Dieses Kapitel wird unter der Creative Commons Namensnennung 4.0 International Lizenz (http://creativecommons.org/licenses/by/4.0/deed.de) veröffentlicht, welche die Nutzung, Vervielfältigung, Bearbeitung, Verbreitung und Wiedergabe in jeglichem Medium und Format erlaubt, sofern Sie den/die ursprünglichen Autor(en) und die Quelle ordnungsgemäß nennen, einen Link zur Creative Commons Lizenz beifügen und angeben, ob Änderungen vorgenommen wurden.

Die in diesem Kapitel enthaltenen Bilder und sonstiges Drittmaterial unterliegen ebenfalls der genannten Creative Commons Lizenz, sofern sich aus der Abbildungslegende nichts anderes ergibt. Sofern das betreffende Material nicht unter der genannten Creative Commons Lizenz steht und die betreffende Handlung nicht nach gesetzlichen Vorschriften erlaubt ist, ist für die oben aufgeführten Weiterverwendungen des Materials die Einwilligung des jeweiligen Rechteinhabers einzuholen.

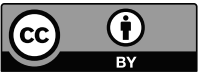

